OPEN ACCESS

Edited by:

Jianke Li,

Institute of Apiculture Research (CAS),

China

Reviewed by:

Beide Fu,

Institute of Hydrobiology (CAS), China

Changwei Shao,

Yellow Sea Fisheries Research

Institute (CAFS), China

${ }^{*}$ Correspondence:

Xuzhen Cheng

chengxuzhen@caas.cn

Lixia Wang

wanglixia03@caas.cn

Specialty section:

This article was submitted to Evolutionary and Population Genetics,

a section of the journal

Frontiers in Genetics

Received: 19 May 2020

Accepted: 11 August 2020

Published: 30 September 2020

Citation:

Wang J, Li J, Liu Z, Yuan X, Wang $S$, Chen $H$, Chen $X$, Cheng $X$ and Wang $L$ (2020) Construction of a

High-Density Genetic Map and Its

Application for QTL Mapping

of Leaflet Shapes in Mung Bean

Nigna radiata $L$.).

Front. Genet. 11:1032.

doi: 10.3389/fgene.2020.01032

\section{Construction of a High-Density Genetic Map and Its Application for QTL Mapping of Leaflet Shapes in Mung Bean (Vigna radiata L.)}

Jie Wang ${ }^{1}$, Jianling Li1,2, Zhenxing Liu ${ }^{3}$, Xingxing Yuan ${ }^{4}$, Suhua Wang ${ }^{1}$, Honglin Chen ${ }^{1}$, Xin Chen ${ }^{4}$, Xuzhen Cheng ${ }^{1 *}$ and Lixia Wang ${ }^{1 *}$

${ }^{1}$ Institute of Crop Sciences, Chinese Academy of Agricultural Sciences, Beijing, China, ${ }^{2}$ College of Life Science, Yangtze University, Jingzhou, China, ${ }^{3}$ Tangshan Academy of Agricultural Sciences, Tangshan, China, ${ }^{4}$ Institute of Industrial Crops, Jiangsu Academy of Agricultural Sciences, Nanjing, China

Mung bean (Vigna radiata L.) is an important but understudied food legume in Asia and now worldwide. Genetic studies may help to accelerate the exploitation of new genes for breeding in this crop. Here, we used a recombination inbred line population to construct an SNP genetic linkage map by genome sequencing technology. We obtained 21,508 high-quality SNP markers integrated into 1,946 bin markers that were mapped onto 11 linkage groups (LGs) with 99-258 bin markers per LG. The total genetic length of the map was $1060.2 \mathrm{cM}(38.76-168.03 \mathrm{cM}$ per LG), with an average distance between markers of $0.54 \mathrm{cM}$. However, there were 18 gaps $>5 \mathrm{cM}$, distribution on LG1, 3, 5, 7, and 9. Gene mapping for lobed and indented leaflets was conducted using the map. A major quantitative trait locus (QTL) associated with indented leaflets was detected on chromosome 10, with phenotypic variation explained (PVE) values of $39.7 \%$ and 45.4\% under two different environments. Several QTLs for lobed leaflets were detected and most of them were tightly linked together on Chromosome 3. However, only one major QTL, which explained the largest phenotypic variation (27.7-69.5\%), was stably detected under two different environments using both $\mathrm{R}$ and $\mathrm{Q}$ methods. In the two main stable QTLs regions on chromosomes 3 and 10, candidate genes for regulating the molecular mechanism of different leaflet shapes were detected by functional annotation. The overlap of major QTLs under different environments indicated that the present map would be good enough for precisely mapping genes, and both the QTL analysis and gene prediction were useful for investigating the mechanism of leaf development in mung bean or legumes.

Keywords: Vigna radiata, high-density map, leaflet shape, QTL, SNP

\section{INTRODUCTION}

Mung bean (Vigna radiata L.), a cultivated species in genus Vigna $(2 \mathrm{n}=2 \times=22)$, has a genome size of about $500 \mathrm{Mb}$ (Yang et al., 2014). It is an important plant protein resource and a potentially healthy food (Nair et al., 2013; Shi et al., 2016) that has traditionally grown across Asia (Tomooka et al., 2002). Mung bean production has spread to most parts of the world in 
recent decades. The worldwide demand of mung bean has greatly increased because of its healthy properties, especially the identification of physiological components in the seeds (Yao et al., 2013), that have attracted much interest. Therefore, breeding new varieties with high yield and high quality is urgently required. Genetic and genomic study can help to improve the efficiency of mining for new genes and breeding of important varieties, but mung bean has been less studied than other major crops.

Linkage map construction and gene mapping are important for genetic and genomic studies. A number of genetic linkage maps for mung bean, based mainly on restriction fragment length polymorphism (RFLPs) (Young et al., 1992; Chaitieng et al., 2002; Humphry et al., 2002) or simple sequence repeats (SSRs) (Kajonphol et al., 2012; Sompong et al., 2012; Wang et al., 2016; Singh et al., 2018), have been reported previously. However, most of these maps were low density because of the limited number of polymorphic markers, although primarily mappings of genes associated with bruchid resistance (Chen et al., 2012; Wang et al., 2016), domesticated traits (Isemura et al., 2012), and agronomic traits (Kajonphol et al., 2012) were reported. With the release of the whole genome sequence of mung bean (Yang et al., 2014), rapid progress on gene tagging and candidate genes associated with resistance to pests such as seed beetles (Coleoptera: Bruchidae) and disease also has been made (Chotechung et al., 2016; Kaewwongwal et al., 2017; Mathivathana et al., 2019). Single nucleotide polymorphism (SNP) maps, essential for precise gene mapping, have been constructed for species related to mung bean, such as black gram and wild cowpea for bruchid resistance (Amkul et al., 2019; Somta et al., 2019), cowpea for yield traits (Pan et al., 2017), and adzuki bean for flowering time (Liu et al., 2016). However, similar studies are rare in mung bean. To enhance the genetic study of mung bean, we constructed an SNP map using a recombinant inbred line (RIL) population. The original parents of the RILs were both cultivated genotypes with different major agronomic traits. To validate the usefulness of the SNP map, we conducted a quantitative trait locus (QTL) analysis of two rare leaflet shapes of mung bean and predicted the candidate genes associated with these traits. The results will help to accelerate the genetic research of mung bean and contribute to the breeding of improved varieties.

\section{MATERIALS AND METHODS}

\section{Plant Material}

Dahuaye, green seeded, is a landrace with lobed leaflet, and Jilyu 9-1, black-seeded, is a natural mutant with indented leaflet that was obtained from field tests, and the indented leaflet was stably inherit after several season's propagation. A cross between these two genotypes in 2012 in Beijing produced a hybrid that was sown in the following season. After harvest of the $F_{1}$ plant, each line of the $F_{2}$ generation was planted continually for several years by the single seed descend method, until an RIL $_{8}$ population was developed in 2017 for high-density map construction.

\section{DNA Preparation, Library Construction, and Sequencing}

The RIL population was continually planted in 2018 and 2019 with 10 seeds for each line. Young leaves of 10 plants from each of 190 randomly selected lines were collected at the 2019 season. DNA was extracted using the CTAB method (Doyle and Doyle, 1987). The quality of the extracted DNA was checked using a NanoPhotometer ${ }^{\circledR}$ spectrophotometer (IMPLEN, Westlake Village, CA, United States) and the concentration was determined using a Qubit ${ }^{\circledR}$ DNA Assay Kit and a Qubit ${ }^{\circledR}$ 2.0 Fluorometer (Life Technologies, Carlsbad, CA, United States).

A total of $1.5 \mu \mathrm{g}$ genomic DNA per sample was fragmented by sonication to $350 \mathrm{bp}$, then end polished, A-tailed, and ligated with the full-length adapter. After PCR amplification, product purification (AMPure XP system, Beckman Coulter, Beverly, MA, United States), and size distribution assessment using an Agilent 2100 Bioanalyzer, the target fragments were quantified by real-time PCR. The prepared libraries were sequenced on an Illumina NovaSeq 6000 PE150 platform (Illumina, San Diego, CA, United States) and 150-bp paired-end reads were generated with insert sizes of about $350 \mathrm{bp}$.

Raw reads in fasta format were processed using in-house C scripts (Biomarker Technologies, Beijing, China) for quality control (QC) to ensure the reliability and that the reads were free of base-calling duplicates, adapter contamination, and other artificial biases. The QC process removed reads with $\geq 10 \%$ unidentified nucleotides, reads with $>50 \%$ low-quality bases, reads with $>10$ nucleotides aligned to the adapter allowing $\leq 10 \%$ mismatches, and putative PCR duplicates generated by PCR amplification in the library construction process.

\section{Mapping to the Reference Genome and SNPs Detection}

The genome sequence of mung bean (GenBank: GCA_000741045.2) was used as the reference genome (Yang et al., 2014). The clean reads in each library were aligned against the reference genome (settings: mem -t $4-\mathrm{k} 32-\mathrm{M}-\mathrm{R}$ ) using the Burrows-Wheeler Aligner (Li and Durbin, 2009). To ensure the accuracy of the SNP detection, duplicates were removed using the Picard Mark Duplicates tool ${ }^{1}$. The local realignment and SNP detection were performed using the Genome Analysis Toolkit (GATK) protocol ${ }^{2}$ (McKenna et al., 2010) according to the position of the clean reads on the reference genome.

\section{Genotyping and Effective Marker Detection}

The SNPs that were polymorphic between the two parents were selected and classified into eight segregation patterns $(\mathrm{ab} \times \mathrm{cd}$, ef $\times$ eg, hk $\times$ hk, lm $\times 1 \mathrm{l}, \mathrm{nn} \times \mathrm{np}$, aa $\times \mathrm{bb}, \mathrm{ab} \times \mathrm{cc}$, and $c c \times a b)$. Only SNPs with the aa $\times$ bb pattern were selected for further analysis. The SNPs with sequencing depth less than 4 were removed. To find recombination breakpoints, we used a

\footnotetext{
${ }^{1}$ http://sourceforge.net/projects/picard/

${ }^{2}$ https://www.broadinstitute.org/gatk/guide/best-practices?bpm=DNAseq\# variant-discovery-ovw
} 
sliding window that contained 15 SNPs to scan the genome, one SNP per slide. The window was typed as aa when the number of aa-type or bb-type in the window was $>11$; in all other cases, the window was typed as ab. After genotyping, the SNPs without recombination were integrated into one bin marker. Markers that had an unusual appearance, low coverage, and/or obvious segregation distortions were filtered prior to linkage analysis. Only the bin markers longer than $10 \mathrm{~Kb}$ were reserved for analysis, and bin markers with abnormal base and segregation distortion $(P<0.01)$ were removed.

\section{Genetic Linkage Map Construction}

The selected bin markers were used to construct the genetic linkage map. The bin markers were divided in linkage groups (LGs) based on their locations on the mung bean reference genome; that is, 11 LGs were preliminary set before mapping. For each LG, the mapping data were formatted according to the instruction of Ici-Mapping. The marker order was inferred nnTwoOpt, in which nearest neighbor and two-opt were used for tour construction and improvement, respectively, and recombinant frequencies were calculated with a minimum logarithm of odd (LOD) threshold of 3. Genetic distances were estimated with the Kosambi's mapping function. Rippling was performed using SARF (sum of adjacent recombination frequencies) with a window size of 5 (Meng et al., 2015).

\section{Observation and Measurement of Leaf Blade Characteristics}

To obtain precise locations of the shapes in the mung bean genome, both the parents and the RILs were investigated for their leaflet shapes under two continually cropping seasons. In the summer of 2018, the RILs were planted in the test site of the Institute of Crop Sciences at the Chinese Academy of Agricultural Sciences (CAAS) $\left(39.96^{\circ} \mathrm{N}\right.$ and $\left.116.33^{\circ} \mathrm{E}\right)$. After harvesting, they were planted in Yazhou $\left(18.38^{\circ} \mathrm{N}\right.$ and $\left.109.19^{\circ} \mathrm{E}\right)$ in winter of 2018 and then in Shunyi $\left(40.23^{\circ} \mathrm{N}\right.$ and $\left.116.56^{\circ} \mathrm{E}\right)$ in summer of 2019. During the flowering stage, a top leaflet of classical ternate compound leaf was collected from the middle part of each plant photographed. For each line, the top leaflets from five plants were evaluated. Because both the lobed and indented leaflets are not classical qualitative traits, to avoid the statistical error in evaluation by visual observation, for the QTL analysis, we counted the number of apexes around each leaf margin for the indented shape, and applied two indexes to evaluate the degree of the lobed leaflet. One index was the ratio of leaf areas, which was calculated using a self-written protocol in Matlab as $R=(\mathrm{V} / \mathrm{A}) \times 100$, where $\mathrm{V}$ is the actual area of the leaflet and $A$ is the sum of the actual and lobed areas. The other index was the ratio of the length and width, which was calculated as $Q=\left(\mathrm{L}_{1} / \mathrm{L}_{2}\right) \times 100$, where $\mathrm{L}_{1}$ is the distance between the intersection of the concave point on both sides of the leaf and the main vein intersection to the base of the leaf, and $L_{2}$ is the distance from the tip to the base of the leaf. Lines with leaflets that showed both lobed and indented characters were removed from the QTL analysis because, when photographed, the curled margins made them difficult to evaluate.

\section{Analysis of QTL and Candidate Genes}

Quantitative trait locus analysis was conducted using the inclusive composite interval mapping (ICIM) method implemented in IciMapping (Meng et al., 2015). $R$ and $Q$ values were used to locate QTL for lobed leaflet and the number of apexes (NA) was used to locate QTL for indented leaflet. The ICIM was performed every $0.1 \mathrm{cM}$ with the probability in stepwise regression set as 0.001 . For the LOD threshold $\mathrm{LOD}=\log _{10}\left(\mathrm{P}_{1} / \mathrm{P}_{2}\right), \mathrm{P}_{1}$ is the probability that the site has a QTL and $\mathrm{P}_{2}$ is the probability that the site has no QTL. Each QTL was determined by a 1000 permutation test at $P=0.05$.

The bin markers were located in the mung bean reference genome using the re-sequencing data. According to the physical location of a bin marker on each side of a QTL, the target region in the reference genome was searched on NCBI website ${ }^{3}$. The genes in the target regions were obtained from the GFF3 file and the KOBAS 3.0 database $^{4}$ was used for the gene ontology (GO) and KEGG (Kyoto Encyclopedia of Genes and Genomes) analysis by inputting the gene list and selecting the "Gene-list enrichment" module. The KEGG pathway was considered to be significantly enriched when the corrected $P$-value was $<0.05$.

\section{RESULTS}

\section{Sequencing and Genotyping}

Totally, $10.99 \mathrm{~Gb}$ and $13.35 \mathrm{~Gb}$ of clean bases for parents Dahuaye and Jilyu9-1, respectively, and $494.03 \mathrm{~Gb}$ for offspring were obtained. The average Q30 for parents and offspring were $92.86 \%$ and $92.19 \%$, and the average GC contents were $34.30 \%$ and $35.18 \%$, respectively. The ratios of mapped clean reads for parents were $95.90 \%$ (Dahuaye) and 95.71\% (Jilyu9-1), and 94.05\% for offspring (Supplementary Table S1).

A total of 564,449 SNP was identified between the parents, and 485,977 (Dahuaye) and 485084 (Jilyu9-1) of them were homozygous. However, only 23,170 of these SNPs had the aa $\times$ bb segregation pattern. These SNPs were further combined into 2202 bin markers for map construction.

\section{Construction of the Genetic Linkage Map}

Among the 190 RIL lines, 15 lines had a fairly high ratio of abnormal SNPs ( $>2 \%$ of the total number of SNPs). Therefore, only 175 lines were used for map construction. After linkage analysis, a total of 1946 bin markers consisting of 21,508 SNPs were finally located onto the 11 LGs. According to the marker positions, there was a good agreement between the LGs and the chromosomes of the mung bean reference genome (Supplementary Table S2). The total length of the new map was $1060.17 \mathrm{cM}$ with an average distance between adjacent markers at $0.54 \mathrm{cM}$. The number of mapped markers in each LG ranged from 99 (LG4) to 258 (LG5) with an average of 177. The average length of the 11 LGs was $96.38 \mathrm{cM}$, LG4 was the shortest $(38.76 \mathrm{cM})$, and LG9 was the longest $(168.03 \mathrm{cM})$. The density of markers on each LG varied greatly with LG2 $(0.35 \mathrm{cM})>$ LG4 $(0.40 \mathrm{cM})>$ LG5

\footnotetext{
${ }^{3}$ https://www.ncbi.nlm.nih.gov/genome/?term=mungbean

${ }^{4}$ http://kobas.cbi.pku.edu.cn/anno_iden.php
} 
$(0.44 \mathrm{cM})>$ LG3 $(0.45 \mathrm{cM})>$ LG7 $(0.48 \mathrm{cM})>$ LG8 $(0.50 \mathrm{cM})>\operatorname{LG} 1(0.51 \mathrm{cM})>\operatorname{LG} 6(0.56 \mathrm{cM})>\operatorname{LG} 10$ $(0.73 \mathrm{cM})>\operatorname{LG} 11(0.74 \mathrm{cM})>$ LG9 $(1.02 \mathrm{cM})$. A total of 18 gaps (>5 cM) were located in LG1, 5, 7, and 9. Seven of the gaps were $1>10 \mathrm{cM}$; four were on LG9 including the longest one $(19.9 \mathrm{cM})$ (Table 1 and Figure 1).

\section{Inheritance of Different Leaflet Shapes}

The leaflets of all $F_{1}$ plants were semi-lobed, and the indented shape did not appear at the first generation (Figure 2), indicating that the lobed leaflet was semi-dominant and the indented shape recessive. Among their offspring, there were individuals that expressed indented and lobed shapes simultaneously (Figure 2), suggesting these two leaflets were not allelic and were inherited independently.

Based on visual observation of the phenotypes, the separation ratio of indented leaflet to ovate shape did not fit 1:1, whereas the separation ratio of lobed leaflet to ovate shape fit $1: 1$ by both $R$ $\left(\chi^{2}=0.0548, P=0.8149\right)$ and $\mathrm{Q}\left(\chi^{2}=1.39, P=0.2386\right)$ methods. Using the precise methods, both the frequency of distribution of both the indented leaflet by counting the number of apexes and the lobed shape using $\mathrm{R}$ and $\mathrm{Q}$ methods did not accord with classical normal distribution, under the 2018 and 2019 environments (Figure 3).

\section{Identification of QTL for the Lobed and Indented Leaflet Traits}

A series of tightly linked peaks on LG3 and LG10 were detected on the LOD contours for the lobed leaflet under the different environments (Figure 4), corresponding with six QTLs on LG3 and two on LG10 (Table 2 and Figure 5). Among them, one major QTL, Block18747-Block 19028 (qLobL.1) spanning $1.0 \mathrm{cM}$ on LG3, was stably detected in the 2018 libraries with $Q$ and $R$ values, with phenotypic variation explained (PVE) values from 50.0 to $69.5 \%$. This stable QTL was physically mapped onto chromosome 3 from 5471015 to 5544796 bp. The other five QTLs on LG3 were identified in the 2019 libraries using $Q$ and $R$ values, with PVE

TABLE 1 | Information of the novel constructed genetic linkage map for mung bean.

\begin{tabular}{lccccc}
\hline LG & $\begin{array}{c}\text { Num. of bin } \\
\text { marker }\end{array}$ & $\begin{array}{c}\text { Total distance } \\
\text { (cM) }\end{array}$ & $\begin{array}{r}\text { Average } \\
\text { distance (cM) }\end{array}$ & $\begin{array}{c}\text { Max gap } \\
\text { (cM) }\end{array}$ & $\begin{array}{c}\text { Num. of gaps } \\
\mathbf{7} \mathbf{5} \text { cM }\end{array}$ \\
\hline LG1 & 223 & 114.27 & 0.51 & 11.4 & 4 \\
LG2 & 209 & 71.90 & 0.35 & 3.2 & 0 \\
LG3 & 101 & 44.55 & 0.45 & 2.6 & 0 \\
LG4 & 99 & 38.76 & 0.4 & 4.4 & 0 \\
LG5 & 258 & 113.37 & 0.44 & 15.8 & 4 \\
LG6 & 229 & 127.99 & 0.56 & 4.4 & 0 \\
LG7 & 202 & 95.76 & 0.48 & 17.9 & 3 \\
LG8 & 223 & 112.09 & 0.5 & 2.9 & 0 \\
LG9 & 165 & 168.03 & 1.02 & 19.9 & 7 \\
LG10 & 121 & 87.85 & 0.73 & 3.8 & 0 \\
LG11 & 116 & 85.60 & 0.74 & 4.5 & 0 \\
Total & 1946 & 1060.17 & 0.54 & - & 18
\end{tabular}

values from 15.8 to $39.8 \%$. These five QTLs were almost connected together from Block19028 to Block19223, with two overlapped confidence intervals from Block18747 to Block18748 (qLobL.2) and Block19219 to Block19223 (qLobL.4). Therefore, one confidence interval from Block18747 to Block18748 (qLobL.2) was the only repeated QTL that could be detected under the two different environments. Two other QTLs for lobed leaflet from Block7476 to Block7482 (qLobL.7) and Block7483 to Block7489 (qLobL.8) on LG10 were detected only in the 2019 libraries, with PVE values of $12.2 \%$ and $15.5 \% 1$, respectively.

For indented leaflet, one common QTL ( $q$ InL.1) on LG10 was detected in the 2018 and 2019 libraries. It was located from Block7460 and Block7463 and spanned about $2.4 \mathrm{cM}$, with PVE values of $39.7 \%$ (2018) and 45.5\% (2019), respectively. QTL $q I n L .1$ was tightly linked with qLobL.7 on LG10.

\section{Candidate Gene Annotation}

We detected 112 genes in all QTL regions related with lobed leaflet, however, only 10 of them were located in the common Block18747-Block18748 region in LG3. Seven of the 10 genes had only non-synonymous SNPs between the parents (Table 3). The products of the homologous genes included polyadenylate-binding protein, late embryogenesis abundant protein, and different protein kinases. The KEGG analysis indicated that LOC106757470 and LOC106757471 were involved in four pathways, namely RNA degradation, ubiquitin-mediated proteolysis, RNA transport, and mRNA surveillance, and that LOC1065757470 was involved in three of these pathways; the exception was ubiquitin-mediated proteolysis.

We detected 45 genes located in the QTL region related with the indented leaflet, and 35 of them encoded homologous products. However, only five had SNPs between the two parents. Four SNPS were in the non-coding regions of LOC106774574, LOC106776045, LOC106774382, and LOC106776095, and seven SNPs were synonymous mutations in LOC106775793. However, the only one SNP could cause an amino acid change, namely tryptophan (Dahuaye) to a stop codon (Jilyu 9-1).

\section{DISCUSSION}

Several maps have been constructed for mung bean with different marker types: RFLP (Young et al., 1992; Lambrides et al., 2000; Humphry et al., 2002), AFLP (Chaitieng et al., 2002), and SSR (Kajonphol et al., 2012; Sompong et al., 2012; Liu et al., 2016; Wang et al., 2016; Singh et al., 2018). The frequent development of SSR had made them popular both in map construction and gene tagging for more than a decade (Han et al., 2005; Wang et al., 2016). However, the densities of these maps were low because of the low level of variation of SSR markers (Wang et al., 2009). SNPs have gradually become more widely used for high density map construction, including in species related to mung bean (Liu et al., 2016; Pan et al., 2017; Amkul et al., 2019; Somta et al., 2019). With the release of the whole genome of 


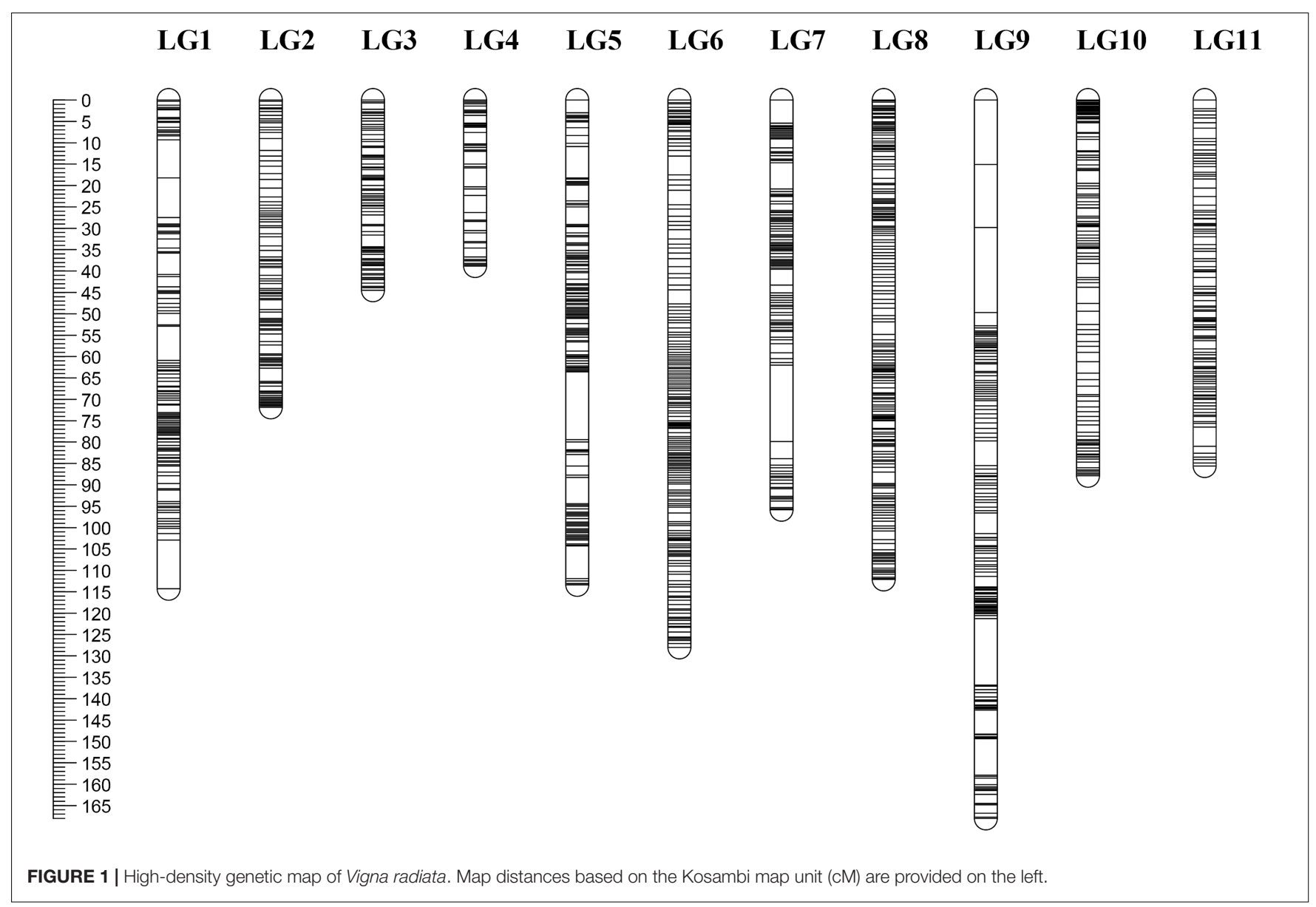

mung bean (Kang et al., 2014), two SNP maps were reported (Schafleitner et al., 2016; Mathivathana et al., 2019). However, because SNP map information is not easily shared among different labs, we constructed our own SNP map.

The order of the markers on LGs in our map agrees well with their locations on the reference genome of mung bean. We consider that the newly constructed map is an improvement over the map constructed by Schafleitner et al. (2016), because in their map, LG3 and LG4 were merged together. The other previous map also agreed well with the reference genome

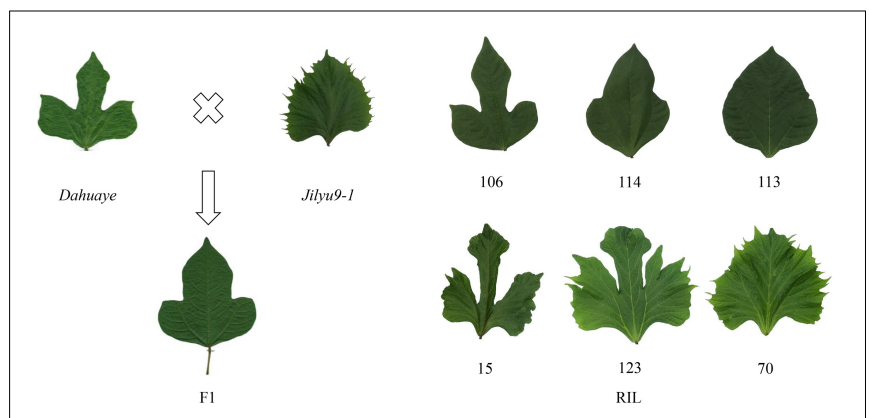

FIGURE 2 | Leaflet shapes of Dahuaye and Jilyu9-1 and their offspring.
(Mathivathana et al., 2019); however, our map has higher density $(0.54 \mathrm{cM})$ than theirs $(3.23 \mathrm{cM})$, mainly because of the different mapping populations and sequencing method.

The total length of our map was much shorter $(1060.2 \mathrm{cM})$ than that of the previous map (1291.7 cM), and the length of the LGs for the two maps also was quite different. For example, in our map, LG9 was the longest (168.03 cM) and LG2 was the shortest $(71.9 \mathrm{cM})$, while in the previous map, LG7 was the longest $(149.1 \mathrm{cM})$ and LG3 the shortest $(90.2 \mathrm{cM})$, whereas in the previous map, LG7 was the longest $(149.1 \mathrm{cM})$ and LG3 the shortest $(90.2 \mathrm{cM})$. In our map, LG9 was the lowest $(1.02 \mathrm{cM})$, followed by LG11 (0.74 cM) and LG10 (0.73 cM), whereas in the previous map, LG8 had the lowest density $(3.03 \mathrm{cM})$, followed by LG10 $(3.02 \mathrm{cM})$ and LG9 $(2.91 \mathrm{cM})$. However, the total trends of the two maps were similar, suggesting that the two maps were in a good agreement. Although the average interval distance was good enough for precise mapping, there were 18 large gaps in our map, especially on LG9, leading to the low density of this LG. Such gaps are seldom found in maps using traditional markers for several main reasons, including that we used a map population derived from two cultivated genotypes, and the genetic backgrounds were more similar than that between a wild and a cultivated genotype. Inaccuracies in the reference genome may be another reason for the gaps in our map. 


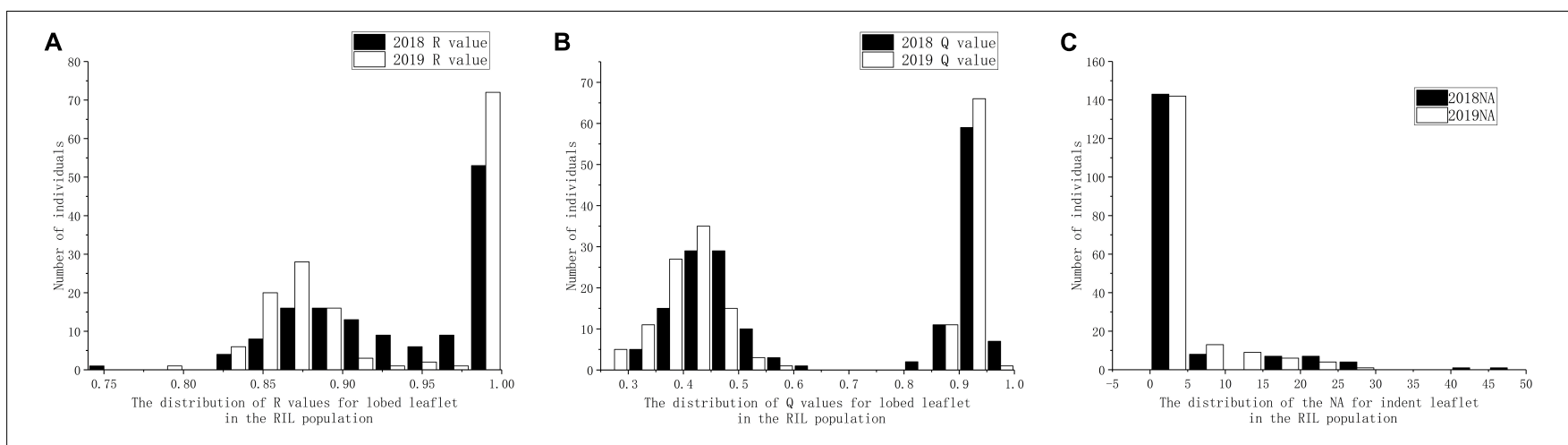

FIGURE 3 | Distribution of leaflet types in the recombinant inbred line (RIL) population derived from Dahuaye $\times$ Jilyu 9-1. (A) Distribution of number of apexes (NA). (B) Distribution of the length ratio $(Q)$ values under different environments. (C) Distribution of the area ratio $(R)$ values under different environments.
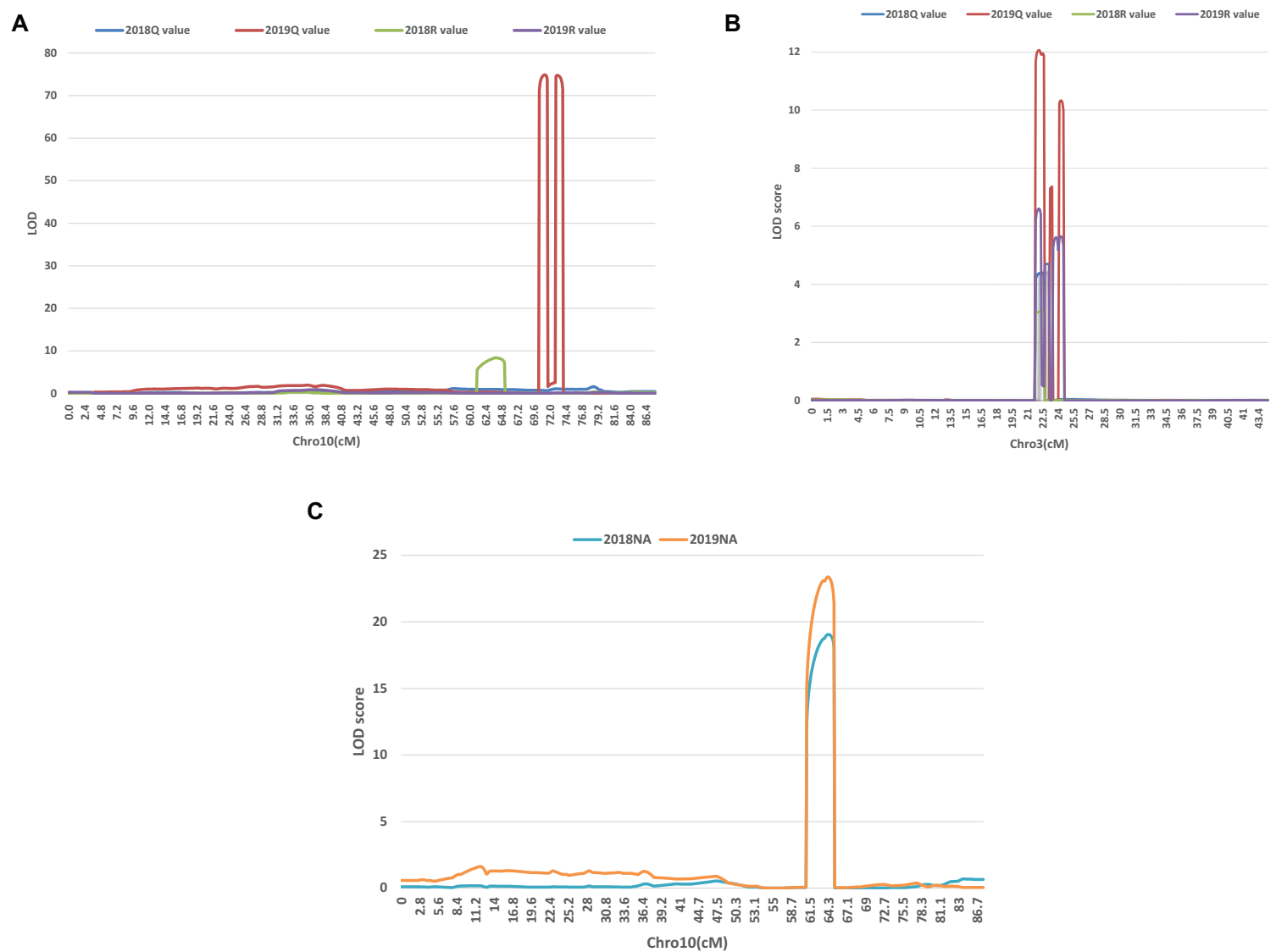

FIGURE 4 | LOD graphs of the QTLs identified for the percentage of lobed leaflet and indented leaflet in a mung bean RIL population of Dahuaye $\times$ Jilyu 9-1 in 2 years (2018 and 2019). (A,B) LOD plots for lobed leaflet. (C) LOD plot for indented leaflet.

To validate the usefulness of the present map, we investigated QTL related with two types of leaflet shapes, lobed and indented. Lobed leaves are common in plants (Zhang et al., 2018), and they also have been reported in mung bean (Jiao et al., 2016). The phenotypes of F1 and F2 plants indicated that the lobed leaflet was semi-dominant and was controlled by multiple genes, and this finding was validated by the QTL analysis. Several
QTLs that were tightly linked together were detected on LG3 and LG10. However, only one was consistently found under different environments both with Q and R methods. This QTL spanned from Block18747 and Block 18748 on LG3, with PVE from 27.7 to $69.5 \%$. The location of this QTL was not overlapped with the other QTLs on our map but was tightly linked with a QTL predicted by Jiao et al. (2016). Whether these two 
TABLE 2 | Description of QTLs associated with the indented and lobed leaflet in mung bean.

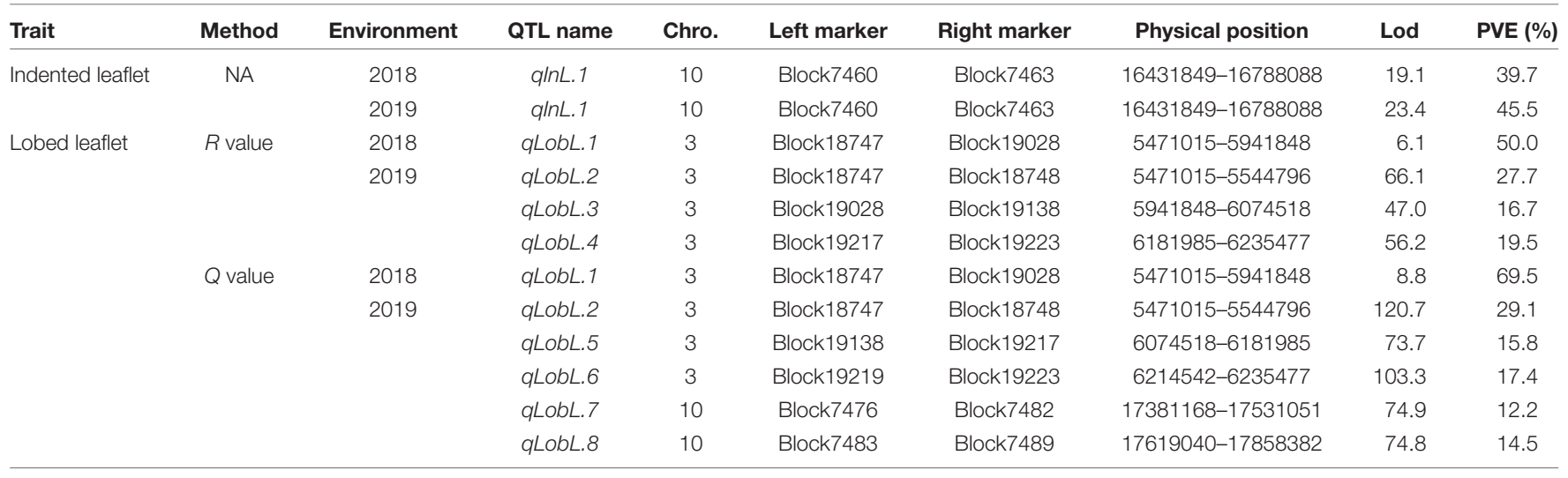

NA, the number of apexes around each leaf margin for the indented shape; PVE, phenotypic variation explained value for a QTL.

\section{LG03}

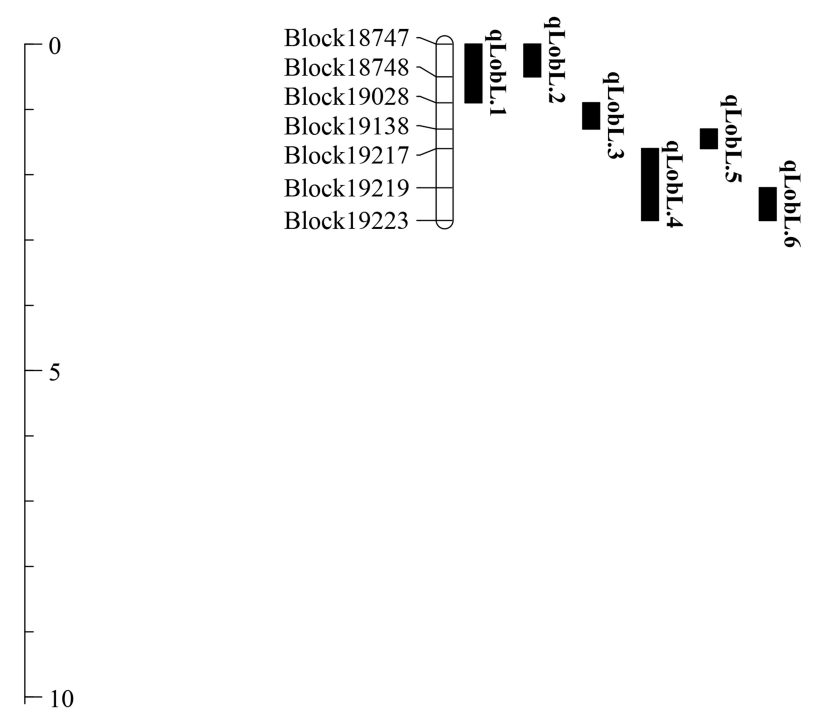

\section{LG10}

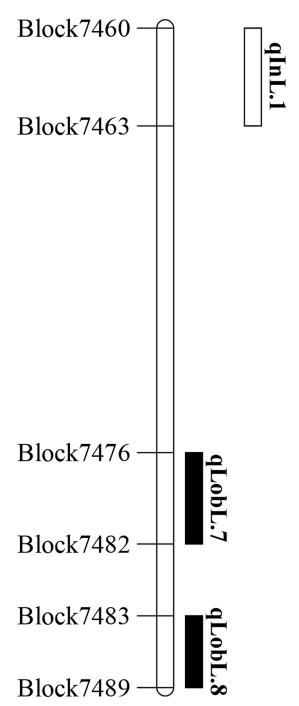

FIGURE 5 | Distribution of QTLs for indented and lobed leaflets in mung bean.

QTL have different allelic genes needs to be further validated. The other QTLs were only found in 2019 library, including two on LG10, indicating these QTLs were easily affected by the environments.

Ten genes were located in the target region of the common QTL related with lobed leaflet and all 10 genes had SNPs between the parents; however, non-synonymous SNPs were found only in seven of these genes. The metabolic pathways involving these genes were mostly unknown, so it was difficult to identify candidate gene associated the development of lobed leaflet. However, overexpression of the gene encoding a glycosyltransferase has been reported to affect the leaf veins in tobacco (Ma et al., 2012), and this gene has been predicted to be homologous with LOC106757416. Therefore, we consider this gene is the most probable candidate gene associated with the lobed leaflet trait.
The indented leaflet is a rare mutant that we found recently in the test field; it has not been reported previously. The indented shape may be caused by the visually observed abnormal variation at the shoot apical meristem, and it also showed differences in

TABLE 3 | SNPs between parents in genes related with lobed leaflet in mung bean.

\begin{tabular}{cccc}
\hline Gene name & Total SNPs & SNPs in exon & Non-synonymous SNPs \\
\hline LOC106757470 & 32 & 14 & 11 \\
LOC106757280 & 11 & 7 & 5 \\
LOC106757335 & 10 & 4 & 3 \\
LOC106757671 & 51 & 8 & 7 \\
LOC106757416 & 12 & 4 & 2 \\
LOC106757422 & 3 & 3 & 1 \\
LOC106757412 & 14 & 4 & 2
\end{tabular}


the number of apexes around the leaflet margins. This trait was considered recessive because it was absent in F1 plants and, based on our observation of F2 plants, it may be controlled by multiple genes. However, we detected only one major QTL spanning Block7460-Block7463 on LG10, and explaining 39.7\% and $45.5 \%$ of PVE under the 2018 and 2019 environments. Although 35 genes with homologous annotations were found within the target region of the major QTL related with indented leaflet, only five genes had 11 SNPs between the parents. Among these SNPs, some were located in non-coding regions and some led to synonymous mutations, only one SNP in the coding region of $L O C 106775793$, resulted in a synonymous mutation. In addition, LOC106775793 was homologous with the gene encoding transcription factor TCP2, which is expressed mainly in rapidly growing tissues or organs and may be related with the development of plant cells (Palatnik et al., 2003; Koyama et al., 2017; Bresso et al., 2018). Overexpression of TCP reduced the degree of indent leaflet (Koyama et al., 2017), and knocked out of TCP led to shrinkage of the leaf margin (Koyama et al., 2010). Therefore, LOC106775793 may be the most probable candidate gene controlling the indented leaflet trait. The QTL analysis not only validated the novel map as useful for fine mapping of other traits but also provides a base for investigating the molecular mechanism controlling leaflet shapes in mung bean and other legumes.

\section{CONCLUSION}

We constructed a high-density genetic linkage map for mung bean using re-sequencing technology, and the map agreed with the reference genome of mung bean. QTLs related with different leaflet shapes were analyzed using this map. One major QTL for indented leaflet was detected on LG10 and several tightly linked QTLs on LG3 and LG10 related with lobed leaflet were detected, but only one of them was consistently detected under different environments with different methods. Genes involved in the molecular mechanism of leaf shape also were predicted. The present map will be useful for fine mapping of genes, and the

\section{REFERENCES}

Amkul, K., Wang, L. X., Somta, P., Wang, S. H., and Cheng, X. Z. (2019). Construction of a high density linkage map and genome dissection of bruchid resistance in zombi pea (Vigna vexillata (L.) A. Rich). Sci. Rep. 9:11719.

Bresso, E. G., Chorostecki, U., Rodriguez, R. E., Palatnik, J. F., and Schommer, C. (2018). Spatial control of gene expression by miR319-regulated TCP transcription factors in leaf development. Plant Physiol. 176, 1694-1708. doi: 10.1104/pp.17.00823

Chaitieng, B., Kaga, A., Han, O. K., Wang, X. W., Wongkaew, S., Laosuwan, P., et al. (2002). Mapping a new source of resistance to powdery mildew in mung bean. Plant Breed. 121, 521-525.

Chen, H. M., Ku, H. M., Schafleitner, R., Bains, T. S., George Kuo, C., Liu, C. A., et al. (2012). The major quantitative trait locus for mung bean yellow mosaic Indian virus resistance is tightly linked in repulsion phase to the major bruchid resistance locus in a cross between mung bean (Vigna radiata (L.) Wilczek) and its wild relative Vigna radiata. Euphytica 192, 205-216. doi: 10.1007/s10681012-0831-9
QTLs and candidate genes will shed light on the development of leaflet shapes in mung bean and other legumes.

\section{DATA AVAILABILITY STATEMENT}

We uploaded the raw data of the re-sequencing to NCBI: PRJNA656124.

\section{AUTHOR CONTRIBUTIONS}

LW conceptualized, designed, supervised the study, and revised the manuscript. JW and JL developed the population and data analysis. ZL, XY, HC, SW, and XiC conducted the data collection. $\mathrm{LW}$ and $\mathrm{XuC}$ secured the research funding. JW wrote the manuscript. All authors read and approved the final manuscript.

\section{FUNDING}

This work was supported by the National Key R\&D Program of China (Nos. 2019YFD1000700 and 2019YFD1000701), the China Agriculture Research System (CARS-08), and the Agricultural Science and Technology Innovation Program (ASTIP) of the Chinese Academy of Agricultural Sciences.

\section{ACKNOWLEDGMENTS}

We thank Liwen Bianji, Edanz Editing China (www.liwenbianji. $\mathrm{cn} / \mathrm{ac}$ ), for editing the English text of a draft of this manuscript. We also thank Dr. Jun Liu from Beijing Normal University for writing the procedure to measure the area of the lobed leaflet.

\section{SUPPLEMENTARY MATERIAL}

The Supplementary Material for this article can be found online at: https://www.frontiersin.org/articles/10.3389/fgene. 2020.01032/full\#supplementary-material

Chotechung, S., Somta, P., Chen, J., Yimram, T., Chen, X., and Srinives, P. (2016). A gene encoding a polygalacturonase-inhibiting protein (PGIP) is a candidate gene for bruchid (Coleoptera: bruchidae) resistance in mung bean (Vigna radiata). Theor. Appl. Genet. 129, 1673-1683. doi: 10.1007/s00122-0162731-1

Doyle, J., and Doyle, J. (1987). A rapid DNA isolation procedure for small quantities of fresh leaf tissue. Phytochem. Bull. 19, 11-15.

Han, O. K., Kaga, A., Isemura, T., Wang, X. W., Tomooka, N., and Vaughan, D. A. (2005). A genetic linkage map for azuki bean [Vigna angularis (Willd.) Ohwi \& Ohashi]. Theor. Appl. Genet. 111, 1278-1287. doi: 10.1007/s00122-0050046-8

Humphry, E., Konduri, V., Lambrides, J., Magner, T., McIntyre, L., Aitken, B., et al. (2002). Development of a mung bean (Vigna radiata) RFLP linkage map and its comparison with lablab (Lablab purpureus) reveals a high level of colinearity between the two genomes. Theor. Appl. Genet. 105, 160-166. doi: 10.1007/s00122-002-0909-1

Isemura, T., Kaga, A., Tabata, S., Somta, P., Srivines, P., Shimiz, T., et al. (2012). Construction of a genetic linkage map and genetic analysis of domestication 
related traits in mung bean (Vigna radiata). PLoS One 7:e41304. doi: 10.1371/ journal.pone.0041304

Jiao, K., Li, X., Guo, W., Yuan, X., Cui, X., and Chen, X. (2016). Genome resequencing of two accessions and fine mapping the locus of lobed leaflet margins in mungbean. Mol. Breed. 36:128.

Kaewwongwal, A., Chen, J., Somta, P., Kongjaimun, A., Yimram, T., Chen, X., et al. (2017). Novel alleles of two tightly linked genes encoding polygalacturonaseinhibiting proteins (VrPGIP1 and VrPGIP2) associated with the Br locus that confer bruchid (Callosobruchus spp.) resistance to mung bean (Vigna radiata) accession V2709. Front. Plant Sci. 8:1692. doi: 10.3389/fpls.2017. 01692

Kajonphol, T., Sangsiri, C., Somta, P., Toojinda, T., and Srinives, P. (2012). SSR map construction and quantitative trait loci (QTL) identification of majr agronomic traits in mung bean (Vigna radiata (L.) Wilczek). SABRAO J Breed. Genet. 44, 71-86. doi: 10.1556/019.70.2019.09

Kang, Y. J., Kim, S. K., Kim, M. Y., Lestari, P., Kim, K. H., Ha, B. K., et al. (2014). Genome sequence of mungbean and insights into evolution within Vigna species. Nat. Commun. 5:5443. doi: 10.1038/ncomms6443

Koyama, T., Mitsuda, N., Seki, M., Shinozaki, K., and Ohme, T. M. (2010). TCP transcription factors regulate the activities of ASYMMETRIC LEAVES1 and miR164, as well as the auxin response, during differentiation of leaves in Arabidopsis. Plant Cell 22, 3574-3588. doi: 10.1105/tpc.110.075598

Koyama, T., Sato, F., and Ohme-Takagi, M. (2017). Roles of miR319 and TCP transcription factors in leaf development. Plant Physiol. 175, 874-885. doi: 10.1104/pp.17.00732

Lambrides, C. J., Lawn, R. J., Godwin, I. D., Manners, J., and Imrie, B. C. (2000). Two genetic linkage maps of mung bean using RFLP and RAPD markers. Aust. J. Agric. Res. 51, 415-425. doi: 10.1071/ar99052

Li, H., and Durbin, R. (2009). Fast and accurate short read alignment with BurrowsWheeler transform. Bioinformatics 25, 1754-1760. doi: 10.1093/bioinformatics/ btp324

Liu, C. Y., Fan, B. J., Cao, Z. M., Su, Q. Z., Wang, Y., Zhang, Z. X., et al. (2016). Development of a high-density genetic linkage map and identification of flowering time QTLs in adzuki bean (Vigna angularis). Sci. Rep. 6:39523. doi: $10.1038 /$ srep39523

Ma, C., Liu, X., Wang, C., Xu, X., Cheng, G., and Tan, Y. (2012). Construction and genetic transformation of glycosyltransferase sm-ngtl deletion expression vector in tobacco. Guangdong Agric. Sci. 39, 146-148.

Mathivathana, M. K., Murukarthick, J., Karthikeyan, A., Jang, W., Dhasarathan, M., Jagadeeshselvam, N., et al. (2019). Detection of QTL associated with mung bean yellow mosaic virus (MYMV) resistance using the interspecific cross of Vigna radiata $\times$ Vigna umbellata. J. Appl. Genet. 60, 255-268. doi: 10.1007/s13353019-00506-x

McKenna, A., Hanna, M., Banks, E., Sivachenko, A., Cibulskis, K., Kernytsky, A., et al. (2010). The genome analysis toolkit: a mapreduce framework for analyzing next-generation DNA sequencing data. Genome Res. 20, 1297-1303. doi: $10.1101 /$ gr.107524.110

Meng, L., Li, H., Zhang, L., and Wang, J. (2015). QTL IciMapping: integrated software for genetic linkage map construction and quantitative trait locus mapping in biparental populations. Crop J. 3, 269-283. doi: 10.1016/j.cj.2015. 01.001

Nair, R. M., Yang, R. Y., Easdown, W. J., Thavarajah, D., Thavarajah, P., Hughes, J., et al. (2013). Biofortification of mung bean (Vigna radiata) as a whole food to enhance human health. J. Sci. Food Agric. 93, 1805-1813. doi: 10.1002/jsfa.6110

Palatnik, J. F., Allen, E., Wu, X., Schommer, C., Schwab, R., Carrington, J. C., et al. (2003). Control of leaf morphogenesis by microRNAs. Nature 425, 257-263. doi: 10.1038/nature01958

Pan, L., Wang, N., Wu, Z., Guo, R., Yu, X., Zheng, Y., et al. (2017). A high density genetic map derived from RAD sequencing and its application in QTL analysis of yield-related traits in Vigna unguiculata. Front. Plant Sci. 8:1544. doi: $10.3389 /$ fpls.2017.01544

Schafleitner, R., Huang, S. M., Chu, S. H., Yen, J. Y., Lin, C. Y., Yan, M. R., et al. (2016). Identification of single nucleotide polymorphism markers associated with resistance to bruchids (Callosobruchus spp.) in wild mung bean (Vigna radiata var. sublobata) and cultivated $V$. radiata through genotyping by sequencing and quantitative trait locus analysis. BMC Plant Biol. 16:159. doi: 10.1186/s12870-016-0847-8

Shi, Z., Yao, Y., Zhu, Y., and Ren, G. (2016). Nutritional composition and antioxidant activity of twenty mung bean cultivars in China. Crop J. 4, 398-406. doi: 10.1016/j.cj.2016.06.011

Singh, N., Mallick, J., Sagolsem, D., Mandal, N., and Bhattacharyys, S. (2018). Mapping of molecular markers linked with MYMIV and yield attributing traits in mung bean. Ind. J. Genet. Plant Breed. 78, 118-126. doi: 10.5958/0975-6906. 2018.00014.7

Sompong, U., Somta, P., Raboy, V., and Srinives, P. (2012). Mapping of quantitative trait loci for phytic acid and phosphorus contents in seed and seedling of mung bean (Vigna radiata (L.) Wilczek). Breed. Sci. 62, 87-92. doi: 10.1270/jsbbs. 62.87

Somta, P., Chen, J., Yundaeng, C., Yuan, X., Yimram, T., Tomooka, N., et al. (2019). Development of an SNP-based high-density linkage map and QTL analysis for bruchid (Callosobruchus maculatus F.) resistance in black gram (Vigna mungo (L.) Hepper). Sci. Rep. 9:3930. doi: 10.1038/s41598-019-40 669-5

Tomooka, N., Vaughan, D., Moss, H., and Maxted, N. (2002). The Asian Vigna: Genus Vigna Subgenus Ceratotropis Genetic Resources. London: Kluwer Academic Publishers.

Wang, L. X., Cheng, X. Z., Wang, S. H., Liu, C. Y., and Liang, H. (2009). Transferability of SSR markers from adzuki bean into mungbean. Acta Agron. Sin. 35, 816-820. doi: 10.1016/S1875-2780(08)60083-8

Wang, L. X., Wu, C. S., Zhong, M., Zhao, D., Mei, L., Chen, H. L., et al. (2016). Construction of an integrated map and location of a bruchid resistance gene in mung bean. Crop J. 4, 360-366. doi: 10.1016/j.cj.2016.06.010

Yang, J. K., Kim, S. K., Kim, M. Y., Lestari, P., and Lee, S. H. (2014). Genome sequence of mung bean and insights into evolution within Vigna species. Nat. Commun. 5:5443. doi: 10.1038/ncomms6443

Yao, Y., Yang, X., Tian, J., Liu, C., Cheng, X., and Ren, G. (2013). Antioxidant and antidiabetic activities of black mung bean (Vigna radiata L.). J. Agric. Food Chem. 61, 8104-8109. doi: 10.1021/jf401812z

Young, N. D., Kumar, L., Menancio-Hautea, D., Danesh, D., Talekar, N. S., Shanmugasundarum, S., et al. (1992). RFLP mapping of a major bruchid resistance gene in mung bean (Vigna radiata L. Wilczek). Theor. Appl. Genet. 84, 839-844. doi: 10.1007/bf00227394

Zhang, Y., Xu, A., Lang, L., Wang, Y., Liu, X., Liang, F., et al. (2018). Genetic mapping of a lobed-leaf gene associated with salt tolerance in Brassica napus L. Plant Sci. 269, 75-84. doi: 10.1016/j.plantsci.2018. 01.005

Conflict of Interest: The authors declare that the research was conducted in the absence of any commercial or financial relationships that could be construed as a potential conflict of interest.

Copyright (c) 2020 Wang, Li, Liu, Yuan, Wang, Chen, Chen, Cheng and Wang. This is an open-access article distributed under the terms of the Creative Commons Attribution License (CC BY). The use, distribution or reproduction in other forums is permitted, provided the original author(s) and the copyright owner(s) are credited and that the original publication in this journal is cited, in accordance with accepted academic practice. No use, distribution or reproduction is permitted which does not comply with these terms. 